\title{
A Quantitative Method for Selection of Enterprise Cloud Computing Models
}

\author{
Dehua YANG \\ School of Economics and Management, Tongji University, Shanghai 200092, China \\ E-mail: yangdh@tongji.edu.cn
}

\begin{abstract}
Targeting at methodological limitations in the decision-making of enterprise cloud computing adoption, this paper analyzes the main influence factors affecting enterprise cloud computing, builds up their hierarchical structures, and works out 8 key influence factors affecting cloud computing models, based on the structural model theory in system engineering. A quantitative method is provided for the selection of optimal or recommended enterprise cloud computing models, in terms of the relationship valuations among key influence factors, cloud computing models, and the factor weights for a specific enterprise.
\end{abstract}

Keywords enterprise cloud computing; structural models; selection of cloud computing models; influence factors of cloud computing

\section{Introduction}

As an emerging technology in information systems development, cloud computing has become very popular for the establishment of enterprise business information systems recently. In the US almost $90 \%$ corporations surveyed were applying or experimenting with cloud computing in their business, in accordance with RightScale report ${ }^{[1]}$ in 2014 . In China, about $50 \%$ corporations surveyed were implementing private clouds according to "China Network World" report last year ${ }^{[2]}$. The fact shows that cloud computing is going to be the leading architecture and technology in enterprise information systems construction.

However, enterprise cloud computing doesn't go smoothly without problems along the way. The cloud computing paradigms should be enterprise specific, because of corporations' differences in both organizational interior and environment. Therefore one of the important issues in enterprise cloud computing applications is how to select the most appropriate cloud computing models for a specific corporation to satisfy its users' requirements, organizational strategies, management practices, and business processes, with which the enterprise managers must confront in the first place.

Enterprise cloud computing models are multidimensional per se. We are still short of in depth study on multidimensional cloud computing models and their relations with enterprise influence factors, as well as on the methods for selecting the best cloud models to help enterprise professionals in their clouds planning and implementation. A lot of studies are still limited to

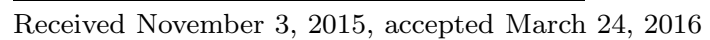


single dimensional models and their influence factors ${ }^{[3-8]}$, such as on service or deployment models, and little attention is paid to an integrated view of the cloud model selection issues. Some researches focus on certain aspects in enterprise business arena ${ }^{[9-13]}$ by exploring the effects of influence factors from certain perspectives including cost-effectiveness, security, cloud providers' services, business models, and sizable corporations, lacking systematic and comprehensive analysis of the main influence factors affecting enterprise cloud computing adoption. For all those contributions, though very valuable and helpful to enterprise cloud computing exploitations, there is no quantitative method put forward to select best cloud computing models.

Targeting at those limitations, the main objective of this article is to provide enterprise management professionals and cloud computing practitioners with a systematic way, by investigating closely the key influence factors and their relations to cloud computing models, to select most appropriate cloud models in enterprise cloud computing adoption. The article works out a paradigm to formulate a quantitative method to deal with these issues, based on the analysis of factors hierarchical structure and the author's own longtime experience in development of enterprise business information systems, in order to assist business professionals and system developers in their cloud computing planning and implementation.

\section{Main Influence Factors Affecting Cloud Computing Models}

Cloud computing models are affected by many influence factors, which are closely related to the characteristics of cloud models. There are many models in enterprise cloud computing from various perspectives, and their characteristics are quite different.

\subsection{Multidimensional Models of Enterprise Cloud Computing}

It seems there is a common viewpoint or consensus among researchers of both academic and business world on two classifications of enterprise cloud models: Service cloud models and deployment models. For service models we have ${ }^{[14-16]}$

- Infrastructure as a service (IaaS),

- Platform as a service (PaaS), and

- Software as a service (SaaS).

For deployment models, we have ${ }^{[16-18]}$

- Public cloud,

- Community cloud,

- Hybrid cloud, and

- Private cloud.

There are a lot of literature discussing those models and their characteristics. These models are formulated from the perspectives of cloud system architecture, composition and shared resource, without consideration of factors in managerial aspects of enterprise, including business strategy, management, security, process and personnel. Therefore those single dimensional 
models (models in one dimension like service or deployment) won't be appropriate in many cases to provide us with an effective paradigm or mechanism to depict a clear and complete picture about cloud computing issues, especially when the enterprise management practice is taken into consideration.

Aiming at those limitations Yang put forward a concept of multidimensional models for enterprise clouds ${ }^{[19]}$. The main idea is to take an integrated view on cloud computing models, as is shown in Figure 1.

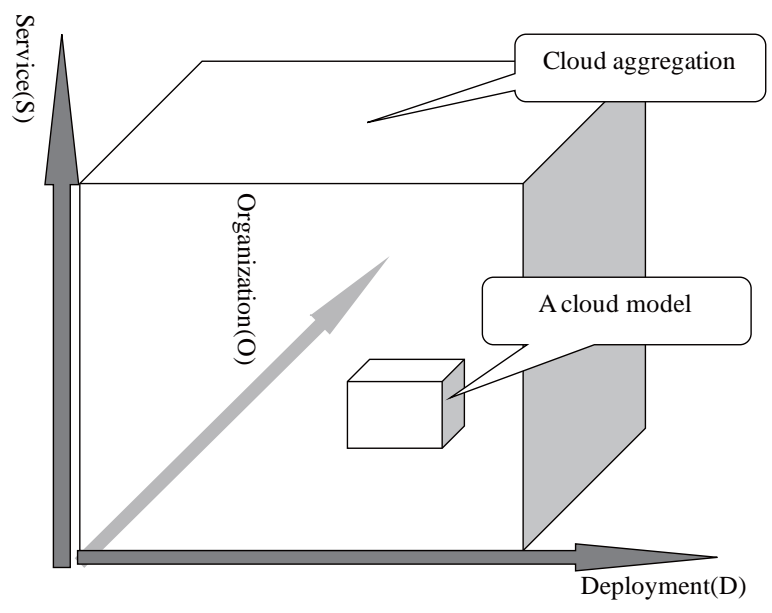

Figure 1 Multidimensional models of enterprise cloud computing ${ }^{[19]}$

In Figure 1 cloud service and deployment are viewed as two dimensions, and a third one is also added: organization dimension, which concerns mainly with enterprise managerial characteristics.

We name the paradigms formulated by combination of service dimension and deployment dimension as 2-dimensional cloud computing models. The 3 -dimensional cloud computing models are the paradigms when the organization dimension is incorporated, as is shown by the cube in the picture. For 2-dimensional cloud models we have 12 paradigms ${ }^{[19]}$ :

- Infrastructure as a service deployed by private cloud (IaaS-Private),

- Platform as a service deployed by private cloud (PaaS-Private),

- Software as a service deployed by private cloud (SaaS-Private),

- Infrastructure as a service deployed by hybrid cloud (IaaS-Hybrid),

- Platform as a service deployed by hybrid cloud (PaaS-Hybrid),

- Software as a service deployed by hybrid cloud (SaaS-Hybrid),

- Infrastructure as a service deployed by community cloud (IaaS-Community),

- Platform as a service deployed by community cloud (PaaS-Community), 
- Software as a service deployed by community cloud (SaaS-Community),

- Infrastructure as a service deployed by public cloud (IaaS-Public),

- Platform as a service deployed by public cloud (PaaS-Public), and

- Software as a service deployed by public cloud (SaaS-Public).

In this article the organization dimension is not used literally. It's only for depiction of influence factors affecting enterprise cloud computing adoption, and how these factors are related to the selection of 2-dimensional cloud models. Namely the enterprise cloud models applied in the selection method are actually two dimensional.

\subsection{Main Influence Factors in Cloud Computing}

What are the influence factors, along the organizational dimension, affecting enterprise cloud computing adoption? To answer this question many authors made a lot of studies on the issue of influence factors from various perspectives. A set of main influence factors is put forward, as shown from Table 1 to Table 5 , based on the analysis of $\operatorname{articles}^{[3-13,16,20-22]}$ and this author's own longtime experience in enterprise business information systems development including cloud computing.

38 influence factors are shown in these tables, which are divided into 5 categories, concerning with cloud systems quality indices, security measures, management practices, resources provided and technological features.

Table 1 Main factors affecting cloud computing: Quality indices

\begin{tabular}{ll}
\hline Factor (No) & Description \\
\hline Usefulness (1) & Satisfy users requirements, be user friendly and easily operational, \\
and have an intuitive interface & Be not too complicated, have structural robustness, be easy to add \\
Maintainability (2) & $\begin{array}{l}\text { functions, debug and upgrade } \\
\text { Be extensible or elastic for applications, processing power, data } \\
\text { storage and network connections }\end{array}$ \\
Flexibility (4) & $\begin{array}{l}\text { Be capable of managing resource and service use in real time, } \\
\text { according to users actual needs } \\
\text { Reliability (5) }\end{array}$ \\
Have low downtime, run smoothly and stably without big \\
performance fluctuations \\
Be capable of integrating with business processes and legacy \\
systems, eliminating info islands \\
Conform to industrial standards, follow business process \\
optimization, and adopt the best practices \\
Have high performance, quick responses, more available functions, \\
Performance (8)
\end{tabular}


Table 2 Main factors affecting cloud computing: Security measures

\begin{tabular}{ll}
\hline Factor (No) & Description \\
\hline Regular security & Have security plan and implementation policy, conduct audits \\
audits (10) & periodically to find, evaluate and cope with potential risks \\
Leaders security & Top leaders are involved in security planning, policy formulation \\
consciousness (11) & and management, have security knowledge and mentality \\
CSO in charge (12) & Conduct chief security officer (CSO) or CIO responsibility system \\
& for security management \\
Incident analysis & Have regulations for security incidents discovering, reporting, \\
and provisions (13) & analysis and countermeasures provisioning \\
Security regulations & Have security guidelines and provisions of using and operating \\
and operations (14) & the system applications, and procedures of coping with contingencies \\
Employee education & Have a policy of employee security related training, educate them \\
$\&$ training (15) & to conform security regulations and rules, esp. for new employees \\
Security standards & Be aware of and conduct security norms and standards, such as \\
adoption (16) & ISO/IEC 27001:2013, GB/T 22081-2008 \\
Data access & Have data protection measures and rules for sensitive information \\
control (17) & accessing, using, and privileges authenticating \\
\hline
\end{tabular}

Table 3 Main factors affecting cloud computing: Management practices

\begin{tabular}{ll}
\hline Factor (No) & Description \\
\hline Top leaders support (18) & $\begin{array}{l}\text { Top leaders support cloud computing adoption, and have certain } \\
\text { knowledge and mentality of business information systems }\end{array}$ \\
Optimization of & Have normalized organization structures and business practices, \\
business processes (19) & like optimizing business processes, for instance, by business \\
& process reengineering (BPR) \\
Collaboration of & Have effective collaborations between various levels of managers \\
departments (20) & and consensus among them towards cloud computing \\
Long run strategic & Have long-term strategic plan for enterprise business \\
plan (21) & informayion systems development and exploitation \\
Project & Have well organized and structured project team, and team \\
management (22) & leaders have sufficient knowledge, experience and competence in \\
& IT systems development \\
Communication and & The related managers/leaders have sufficient ability of \\
users involvement (23) & articulation for new systems adoption, getting employees \\
& understood and involved in the project \\
\hline
\end{tabular}

It seems not practical to use all 38 influence factors in the analysis and selection of cloud computing models, especially for enterprise managers or management professionals. To take them all into account is even not necessary or helpful in most cases, because it could blur the key issues in the adoption of cloud computing and make us distracted by those trivial ones. Therefore, we should simplify and reduce these influence factors and find the most important ones, or the key influence factors, in order to provide enterprise planners and implementers with 
a simple, intuitive and formal way for the decision of selecting best cloud computing models.

Table 4 Main factors affecting cloud computing: Resources provided

\begin{tabular}{ll}
\hline Factor (No) & Description \\
\hline Developers (24) & Have knowledge of software engineering, skills and experience \\
& in business information systems development \\
Time schedule (25) & Time availability for cloud systems development and deployment \\
Project budgets (26) & Budgets availability for the cloud computing adoption project \\
Systems development & The availability of related hardware, software, networks and \\
environment (27) & documents for the cloud computing systems development \\
Cloud providers & Cloud providers reputation, their strategic and management practice, \\
credibility (28) & and their accountability \\
Cloud providers & Cloud services provided by providers, their competitive advantages \\
services (29) & for the services going to be applied \\
Network facilities (30) & Cloud providers have sufficient network resources for customers \\
& like bandwidth, IP addresses, routing paths, and so on \\
Favorite from cloud & Cloud providers extra or preferential services including licensing, \\
providers (31) & security facilities, and other specialties \\
\hline
\end{tabular}

Table 5 Main factors affecting cloud computing: Technological features

\begin{tabular}{ll}
\hline Factor (No) & Description \\
\hline On-demand self-service (32) & Consumer can unilaterally customize computing capabilities, \\
& such as server time, network storage and applications \\
Broad network access (33) & Capabilities are available over the network that can be \\
& accessed through standard mechanisms and heterogeneous \\
& client platforms \\
& The providers computing resources are pooled to serve \\
Resource pooling (34) & multiple consumers, with different physical and virtual \\
& resources dynamically assigned and reassigned to them \\
& according to consumer demand \\
& Capabilities can be elastically provisioned and released, in \\
& some cases automatically, to scale rapidly outward and \\
Rapid elasticity (35) & inward conforming to demand \\
& Cloud systems automatically control and optimize resource \\
use by leveraging a metering capability at some level & appropriate to the type of service \\
Measured service (36) & Security investment on technologies is allocated including \\
the adoption of security applications such as firewall, & encryption, intruder detection, and access control \\
Security technology & backup systems, to keep the systems up to date and alleviate \\
applications (37) & security holes, performance regressions, and other issues \\
and patching (38) &
\end{tabular}

Not all these influence factors are independent and they are related in some way, if we take a close look at their relations. Some factors are dominant comparing to others, and certain 
factors are only effective when others are in position and effective. For example, top leaders support is dominant in undertaking cloud computing, and employees security training is only possible when top leaders have security consciousness. Therefore, if we manage to have a way to find out the most important factors that have dominant positions, control roles and more impact on factors effectiveness, those factors could be viewed as key influence factors and used as arguments for the analysis in selection of cloud computing models, while the other factors could just be omitted safely without losing the expected rationality and usefulness in question. The structural model theory in system engineering can serve as a tool to deal with that issue to simplify and reduce the influence factors.

\section{Structural Model of Main Influence Factors}

A model, which will help to find out the key influence factors in cloud computing adoption and serve as a quantitative method, is worked out by using system engineering theory, in terms of influence factors' hierarchical structure. The basic hypothesis is that the factors in higher levels are more influential and effective than lower level ones, because they have more impact on factor system.

\subsection{System Structural Model}

A digraph representing all influence factors, expressed by a set $F$, and their dependencies can be drawn up. $F$ has $n$ elements corresponding to all influence factors.

$$
F=\left[F_{1}, F_{2}, \cdots, F_{n}\right]
$$

We can establish the adjacency matrix $A$ expressing factor dependencies in accordance with the digraph, and its reachability matrix can be calculated by

$$
R=I \cup A \cup A^{2} \cup \cdots \cup A^{n}=(I \cup A)^{n},
$$

where $I$ is a $n \times n$ identity matrix, $A$ and $R$ are Boolean matrices.

The hierarchical structures of the factor system can be formulated, in accordance with the reachability matrix $R^{[23]}$. Suppose $F_{i}$ 's reachable set is $R S\left(F_{i}\right)$, and its antecedent set is $A S\left(F_{i}\right)$, then for factors at the lowest level, we have

$$
R S\left(F_{i}\right)=R S\left(F_{i}\right) \cap A S\left(F_{i}\right) .
$$

So we can get the lowest level factors according to Equation (3). Then these factors are discarded temporarily from the factor system, and we compute the rest factors with the same process again to get next lowest level factors. Suppose the factor sets at different levels are expressed by $L_{1}, L_{2}, \cdots, L_{k}$, and $L_{0}=\Phi$, we have

$$
L_{j}=\left\{F_{i} \in F-F_{0}-F_{1}-\cdots-L_{j-1} \mid R S_{j-1}\left(F_{i}\right) \cap A S_{j-1}\left(F_{i}\right)=R S_{j-1}\left(F_{i}\right)\right\},
$$

where $j=1,2, \cdots, k, k$ is number of the structure levels. To continue the computation according to Equation (4), until all factors are discarded, namely $L_{0}=\Phi$ is reached, then we have all factor sets at different levels.

$$
\prod_{2}(F)=\left[L_{1}, L_{2}, \cdots, L_{k}\right]
$$


We select certain levels of factors from bottom (the highest level) up as the key factors of the factor system. For example, we select $h$ levels starting from bottom, then the key factors are

$$
\prod_{2}^{\mathrm{Key}}(F)=\left[L_{k}, L_{k-1}, \cdots, L_{k-h+1}\right]
$$

\subsection{The Key Influence Factors Affecting Adoption of Cloud Computing Models}

If we analyse the relations between influence factors put forward in Section 2.2, a digraph can be formulated as Figure 2 (the numbers represent corresponding factors, please refer to Tables 1 to 5). The dependencies are drawn from a project including 3 students under author's instruction, and verified by consulting with 4 management professionals (conducted in a survey, see section 4.1).



Figure 2 Digraph of influence factors

Then we can obtain the adjacency matrix $A$, shown in Figure 3, and calculate the reachability matrix $R$ by Equation (2), as shown in Figure 4. Because there is no strong coupling between factors, and all links are unilateral, so we have

$$
R S\left(F_{i}\right) \cap A S\left(F_{i}\right)=F_{i}
$$

According to Equation (4), Equation (7), and the procedures described in section 3.1, we can get all levels of influence factors. The result is as follows. 


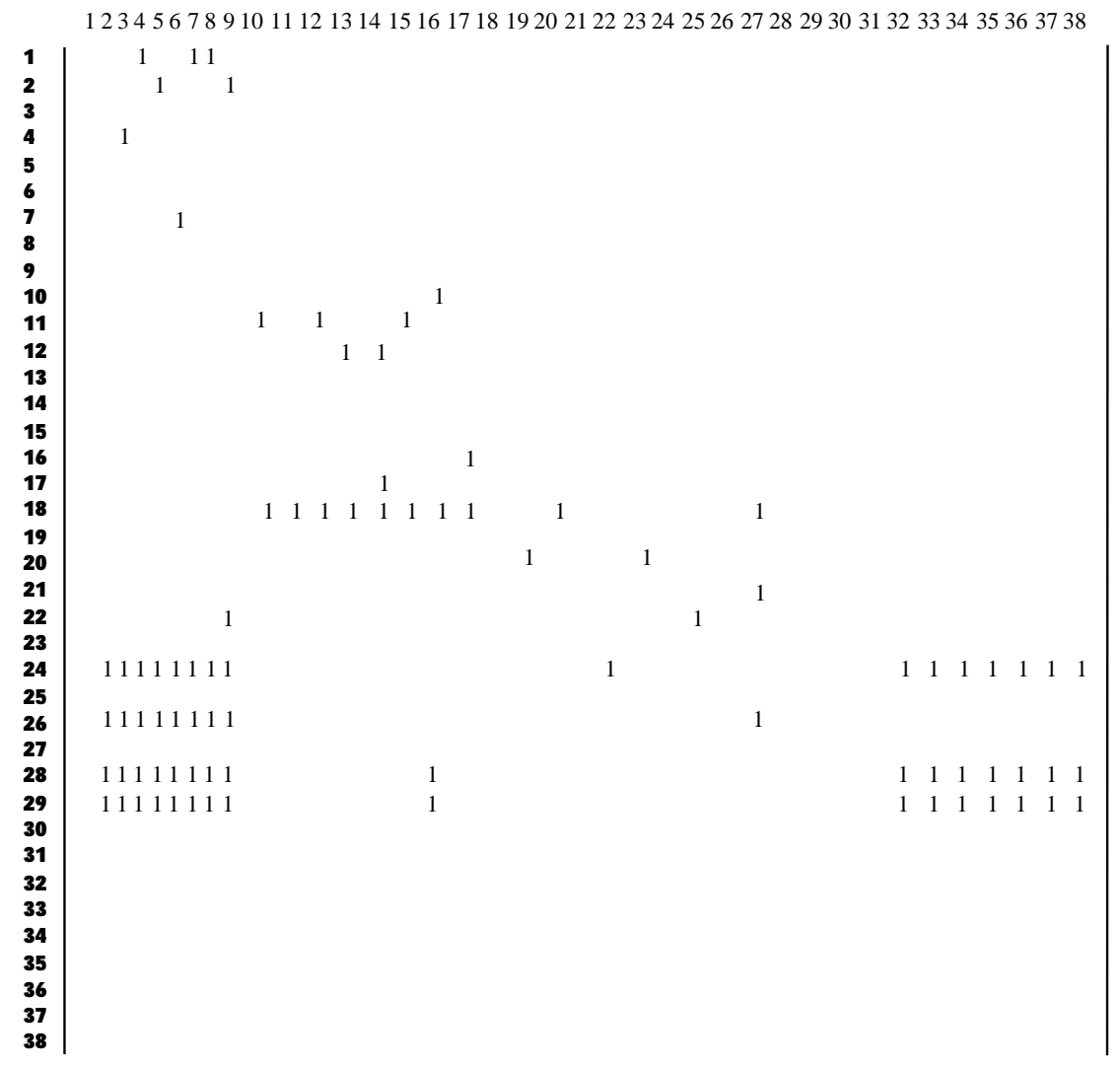

Figure 3 Adjacency matrix $(A)$ of influence factors

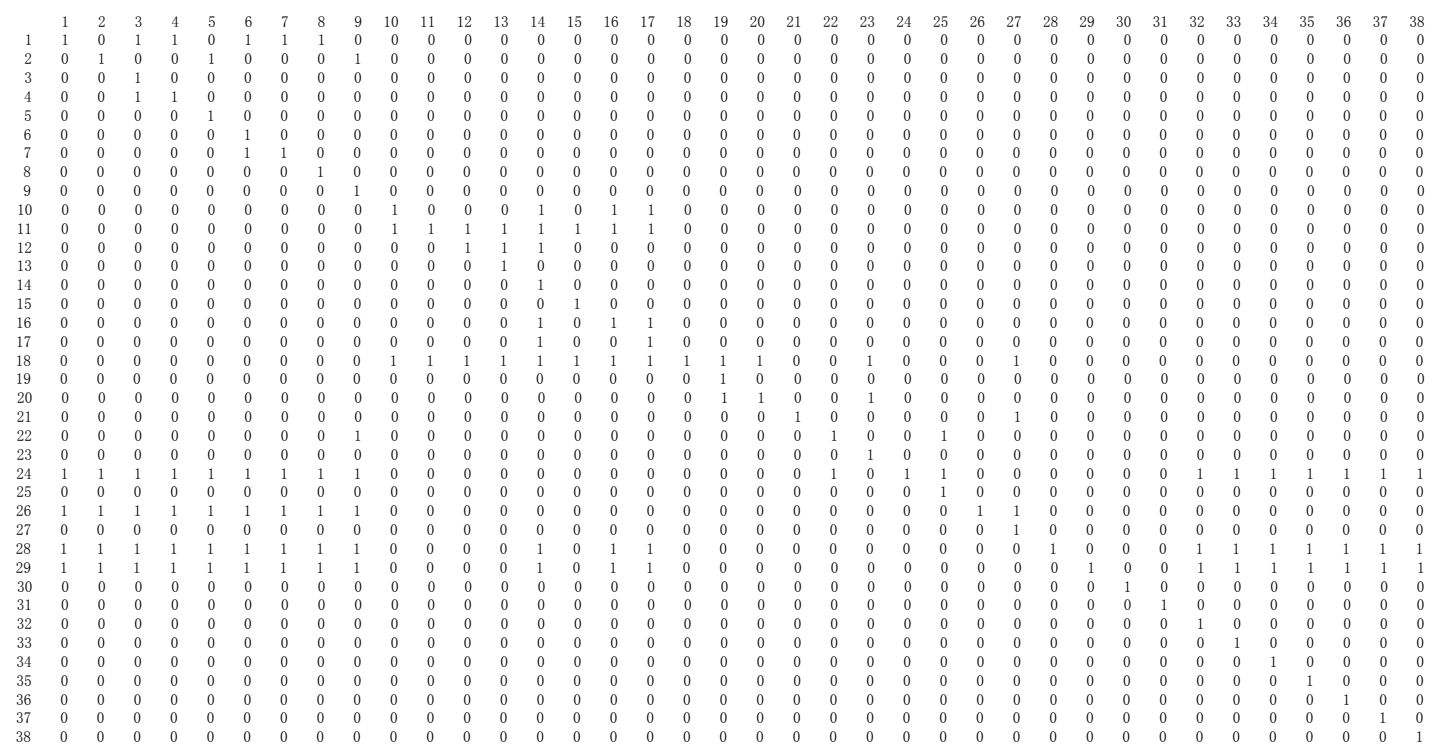

Figure 4 Reachability matrix $(R)$ of influence factors 


$$
\begin{aligned}
L_{1}= & {\left[F_{3}, F_{5}, F_{6}, F_{8}, F_{9}, F_{13}, F_{14}, F_{15}, F_{19}, F_{23}, F_{25}, F_{27}, F_{30}, F_{31},\right.} \\
& \left.F_{32}, F_{33}, F_{34}, F_{35}, F_{36}, F_{37}, F_{38}\right], \\
L_{2}= & {\left[F_{2}, F_{4}, F_{7}, F_{12}, F_{16}, F_{17}, F_{20}, F_{21}, F_{22}\right], } \\
L_{3}= & {\left[F_{1}, F_{10}, F_{24}, F_{26}, F_{28}, F_{29}\right], } \\
L_{4}= & {\left[F_{11}\right], \quad L_{5}=\left[F_{18}\right] . }
\end{aligned}
$$

Commonly by thumb rule we can select no more than 10 influence factors as the key influence factors ( $7 \pm 2,7$ is the "magic number"). The factors in the last 3 levels are selected as the key factors, so we have 8 key influence factors in all.

$$
\prod_{2}^{k e y}(F)=\left[L_{5}, L_{4}, L_{3}\right]=\left[F_{18}, F_{11}, F_{1}, F_{10}, F_{24}, F_{26}, F_{28}, F_{29}\right] .
$$

\subsection{Comments on the Key Influence Factors in Cloud Computing}

In accordance with Table 1 to Table 5 and Equation (8), the key influence factors are

$F_{18}$ : Top leaders support,

$F_{11}$ : Leaders security consciousness,

$F_{1}$ : Usefulness,

$F_{10}$ : Regular security audits,

$F_{24}$ : Developers,

$F_{26}$ : Project budgets,

$F_{28}$ : Cloud providers creditability, and

$F_{29}$ : Cloud providers services.

We know from the factor descriptions that the key influence factors concern mainly with

- Enterprise top managers mentality and involvement in cloud computing adoption,

- Cloud computing systems to satisfy users requirements,

- The knowledge, skills and competence of developers and available budgets,

- Enterprise security regulations and practices, and

- Cloud providers accountability and service quality.

Most key influence factors are related to enterprise management practices. We could draw a conclusion from the results that the enterprise adoption of cloud computing is actually more a problem of enterprise business management, rather than that of application technology, from the viewpoint of key influence factors.

\section{Selection of Enterprise Cloud Computing Models}

There are 12 2-dimensional cloud models as we discussed in section 2.1. But which models are best or most suitable for an enterprise? We can answer this question if we know the relationships between enterprise cloud computing influence factors and the cloud models, as well as the weights of those factors for a specific corporation. 


\subsection{The Relationships Between Cloud Models and Influence Factors}

The relationships between cloud models and influence factors are enterprise specific. But there is some kind of stability or consistency in the relationships revealed by a survey conducted by a project instructed by the author, regarding to what are the effects of key influence factors on cloud computing models. There are 28 professionals (high profile seniors relatively) from 5 corporations surveyed by questionnaires and interviews, the survey profile is described as Table 6. In the survey the management professionals were mainly interviewed, and their questionnaires were filled with help of the survey project members, because of their lacking IT specific knowledge. At the meantime we also consulted them with dependencies among influence factors.

Table 6 Profile of the survey

\begin{tabular}{lll}
\hline Profession & Number & Form of survey \\
\hline Top level manager & 2 & 2 Interviews \\
Department leader & 4 & 2 Questionnaires, 2 interviews \\
System analyst and software engineer & 22 & 22 Questionnaires (3 not valid) \\
\hline
\end{tabular}

We got 21 valid questionnaires having questions regarding how strong are the 8 key influence factors affecting enterprise models, with Likert scales (5 to 1): Very strong, strong, medium, certain, and small. The results (averages rounded to integers) are summarized as relationship matrix in Table 7 (The 4 interviews were evaluated by the team based on their talks).

Table 7 The relationship matrix for 2-dimensional cloud models and key influence factors

\begin{tabular}{lllllllll}
\hline Model/Factor & $F_{18}$ & $F_{11}$ & $F_{1}$ & $F_{10}$ & $F_{24}$ & $F_{26}$ & $F_{28}$ & $F_{29}$ \\
\hline IaaS-Private & 1 & 5 & 5 & 5 & 2 & 2 & 1 & 1 \\
PaaS-Private & 2 & 4 & 5 & 4 & 3 & 3 & 1 & 1 \\
SaaS-Private & 2 & 3 & 4 & 4 & 4 & 4 & 1 & 1 \\
IaaS-Hybrid & 2 & 5 & 5 & 5 & 3 & 2 & 2 & 2 \\
PaaS-Hybrid & 3 & 4 & 5 & 4 & 4 & 3 & 2 & 2 \\
SaaS-Hybrid & 3 & 3 & 4 & 4 & 5 & 4 & 2 & 3 \\
IaaS-Community & 3 & 5 & 4 & 4 & 3 & 3 & 2 & 3 \\
PaaS-Community & 3 & 4 & 3 & 3 & 4 & 4 & 3 & 3 \\
SaaS-Community & 4 & 3 & 2 & 3 & 5 & 5 & 4 & 4 \\
IaaS-Public & 4 & 4 & 4 & 4 & 4 & 3 & 3 & 4 \\
PaaS-Public & 5 & 3 & 3 & 3 & 5 & 4 & 4 & 5 \\
SaaS-Public & 5 & 2 & 2 & 2 & 3 & 5 & 5 & 5 \\
\hline
\end{tabular}

\subsection{Selection of Cloud Models}

Here is an example showing how to use the relationship matrix for best cloud models selection. For a specific enterprise the key influence factors' importance or weight can be 
evaluated by relevant experts, management professionals or consultants. Suppose the vector of key influence factor weights $W$ is

$$
W=\left[W_{1}, W_{2}, W_{3}, W_{4}, W_{5}, W_{6}, W_{7}, W_{8}\right]^{\mathrm{T}},
$$

where $W_{1}$ to $W_{8}$ correspond to $F_{18}, F_{11}, F_{1}, F_{10}, F_{24}, F_{26}, F_{28}$, and $F_{29}$. Suppose the relationship matrix is $C$, which is $12 \times 8$ matrix having values taken from Table $7, A V()$ means evaluation function, $C M$ is a vector of 12 cloud models, then we have

$$
A V(C M)=\left|\begin{array}{r}
A V \text { (IaaS }- \text { Private }) \\
A V \text { (PaaS }- \text { Private }) \\
A V \text { (SaaS }- \text { Private }) \\
A V \text { (IaaS }- \text { Hybrid }) \\
A V(\text { PaaS }- \text { Hybrid }) \\
A V \text { (SaaS }- \text { Hybrid }) \\
A V \text { (IaaS }- \text { Community }) \\
A V \text { (PaaS }- \text { Community }) \\
A V \text { (SaaS }- \text { Community }) \\
A V \text { (IaaS }- \text { Public }) \\
A V \text { (PaaS }- \text { Public }) \\
A V \text { (SaaS }- \text { Public })
\end{array}\right|=C * W .
$$

Suppose the vector of best or recommended cloud models are in $C M_{\mathrm{opt}}$, which have maximum value of $A V()$, and can be obtained by the following equation:

$$
C M_{\mathrm{opt}}=C M_{m} \mid A V\left(C M_{m}\right)=\max \left(A V\left(C M_{i}\right)\right) .
$$

For example, a corporation has weights vector

$$
W=[3,4,4,3,3,4,5,5]^{\mathrm{T}} .
$$

The best or recommended cloud model(s) can be found by Equation (9) and Equation (10).

$$
\begin{aligned}
& A V(C M)=C * W=[82,85,84,98,101,105,103,104,116,115,124,116]^{\mathrm{T}}, \\
& C M_{\mathrm{opt}}=[\mathrm{PaaS}-\text { Public }] .
\end{aligned}
$$

Namely, the best cloud model for the corporation is "platform as a service deployed by public cloud" in the enterprise cloud computing adoption.

\subsection{Some Comments and Discussions}

There are some limitations about the evaluation data in Table 3. The sample is relative small, and the survey is limited to small and medium size enterprises (SME) with low maturity on IT applications in China. For sizable corporations, or some corporations with high level of IT internal drivers or special demand on some aspects of cloud computing systems, such as more security and high performance, the data in Table 3 may not be appropriate or some of them even 
not correct. For different categories of enterprises we could have different relationship matrices, which would be drawn up as needed in the planning stage of cloud computing adoption.

Another issue is that in some special cases certain important influence factors may be omitted, because they are in lower levels. To deal with this issue we could add those omitted important factors to the key influence factor set and reformulate and reevaluate the relationship matrix accordingly.

The quantitative method is mainly a theoretical exploration, empirical studies could be also needed to show its effectiveness in practice.

\section{Conclusions}

Enterprise cloud computing adoption is affected by many factors. The importance of different factors to enterprise cloud models is different, therefore we can find the most important or key influence factors in terms of their impact on the factor system by structural models in system engineering.

This article puts forward a relative formal and quantitative method for enterprise professionals to select most appropriate cloud computing models for their business. At first, we find out the main influence factors in cloud computing, through multidimensional cloud models with the concept of organizational dimension, extensive literature reviews, and our experience. Next, a structural model expressing factor system hierarchy is drawn up, by which we figure out the key influence factors by choosing certain highest levels of factors. Finally, we work out a quantitative method to select the best or recommended cloud models, by means of the relationship matrix representing the factors effectiveness or strength on cloud models and the key influence factor weights expressing factors' importance to a specific corporation.

\section{References}

[1] RightScale. RightScale 2014 State of the Cloud Report. RightScale Inc: (2014-04-02).

[2] Zhou Y. The report of situations and requirements on enterprise cloud computing applications in 2014. China Network World, July 10 2014. http: //cloudgo.cnw.com.cn/cloud-original/htm2014/ 20140710_306166.shtml (in Chinese).

[3] Abdollahzadehgan A, Hussin A R, Gohary M M, et al. The organizational critical success factors for adopting cloud computing in SMEs. Journal of Information Systems Research and Innovation (JISRI), 2013, 4(1): 67-74.

[4] Garrison G, Kim S, Wakefield R L. Success factors for deploying cloud computing. Communications of the ACM, September, 2012, 55(9): 62-68.

[5] Khurana S, Verma A G. Comparison of cloud computing service models: SaaS, PaaS, IaaS. International Journal of Electronics \& Communication Technology, April-June, 2013, 4(3): 29-32.

[6] Parhizkar P, Abdulhussein A N, Joshi J H, et al. A common factors analysis on cloud computing models. International Journal of Computer Science Issues, 2013, 10(2): 523-520.

[7] Subashini S, Kavitha V. A survey on security issues in service delivery models of cloud computing. Journal of Network and Computer Applications, 2011, 34(1): 1-11.

[8] Wu W, Lan L W, Lee Y. Exploring decisive factors affecting an organization's SaaS adoption: A case study. International Journal of Information Management, 2011, 31(6): 556-563.

[9] Bogataj K, Pucihar A. Business model factors influencing cloud computing adoption: Differences in opinion. 26th Bled eConference eInnovations: Challenges and Impacts for Individuals, Organizations and Society, Bled, Slovenia, June 9-13, 2013, 443-455.

[10] Lian J, Yen D C, Wang Y. An exploratory study to understand the critical factors affecting the decision to 
adopt cloud computing in Taiwan hospital. International Journal of Information Management, 2014, 34(1): 28-36.

[11] Montemayor A D, Lopez J F, Wlvarez J C. Critical factors affecting the utilization of cloud computing. CBU International Conference on Innovation, Technology Transfer and Education, Prague, Czech Republic, February 3-5, 2014, 331-336.

[12] Nasina J, Tripathi S. A review of factors that affect cloud computing adoption. The IUP Journal of Computer Sciences, 2013, 7(4): 48-59.

[13] Youssef A E. Exploring cloud computing services and applications. Journal of Emerging Trends in Computing and Information Sciences, 2012, 3(6): 838-847.

[14] Armbrust M, Fox A, GRiffith R, et al. A view of cloud computing. Communications of the ACM, 2010, 53(4): $50-58$.

[15] Marston S, Li Z, Bandyopadhyay S, et al. Cloud computing — The business perspective. Decision Support Systems, 2011, 51(1): 176-189.

[16] Mell P, Timothy G. The NIST definition of cloud computing. National Institute of Standards and Technology, 2011.

[17] Bojanova I, Adelph I C. Analysis of cloud computing delivery architecture models. 25th IEEE International Conference on Advanced Information Networking and Applications Workshops (WAINA 2011), Singapore, March 22-25, 2011, 453-458.

[18] Rahimli A. Factors influencing organization adoption decision on cloud computing. International Journal of Cloud Computing and Services Science (IJ-CLOSER), 2013, 2(2): 140-146.

[19] Yang D. Multidimensional models of enterprise cloud computing. International Conference on Advances in Management Engineering and Information Technology (AMEIT 2015), Bangkok, Thailand, June 28-29 2015, 366-372.

[20] Legris P, Ingham J, Collerette P, et al. Why do people use information technology? A critical review of the technology acceptance model. Information and Management, January 2003, 40(3): 191-204.

[21] Mahmood M A, Burn J M, Gemoets L A, et al. Variables affecting information technology end-user satisfaction: A meta analysis of the empirical literature. International Journal of Human Computer Studies, 2000, 52(4): 751-771.

[22] Seddon P B, Calvert C, Yang S. A multi-project model of key factors affecting organizational benefits from enterprise systems. MIS Quarterly, 2010, 34(2): 305-328.

[23] Wang Z T. Systems Structural Models. In Introduction to System Engineering. Beijing: Electronic Industry Press, 1984 (in Chinese). 